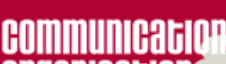
Organlofidin.
Communication et organisation

Revue scientifique francophone en Communication

organisationnelle

$50 \mid 2016$

Engagement entrepreneurial et territoires

\title{
Pour une perspective communicationnelle et pratique de la compétence collective
}

For a practical and communicational perspective of collective competence

\section{Nicolas Arnaud}

\section{OpenEdition}

\section{Journals}

Édition électronique

URL : http://journals.openedition.org/communicationorganisation/5443

DOI : 10.4000/communicationorganisation.5443

ISBN : 979-10-300-0127-3

ISSN : $1775-3546$

Éditeur

Presses universitaires de Bordeaux

Édition imprimée

Date de publication : 1 décembre 2016

Pagination : 215-244

ISBN : 979-10-300-0069-6

ISSN : 1168-5549

Référence électronique

Nicolas Arnaud, «Pour une perspective communicationnelle et pratique de la compétence collective », Communication et organisation [En ligne], 50 | 2016, mis en ligne le 01 décembre 2019, consulté le 16 février 2021. URL : http://journals.openedition.org/communicationorganisation/5443 ; DOI : https:// doi.org/10.4000/communicationorganisation.5443 


\title{
Pour une perspective communicationnelle et pratique de la compétence collective
}

\author{
Nicolas Arnaud'
}

Du fait dela complexité de nos environnements, un des enjeux contemporains de nos entreprises est celui de la recomposition permanente de l'organisation, des processus et des pratiques à l'interface des métiers, des équipes, des départements, voire des relations de collaboration interorganisationnelles. Pour les entreprises, répondre à un tel enjeu nécessite de prendre sérieusement en considération la dimension collective tant de l'action que des connaissances et des compétences (Arnaud 2011, Koenig 1994). Cela constitue une condition, voire un impératif, pour assurer a minima leur pérennité et au-delà leur développement dans l'environnement actuel, nécessitant davantage de flexibilité, de rapidité, d'efficacité et d'efficience (D'Aveni 1995).

En se positionnant à l'interface des travaux en théorie des organisations et en Gestion des Ressources Humaines, cet article soutient qu'une manière pertinente de se saisir de cet enjeu implique d'étudier l'entremêlement des pratiques et de la communication au service de la compétence collective (CC). Considérant que la CC neémerge jamais de façon harmonieuse et instantanée, qu'il faut du temps pour la construire et que les conflits, c'est-à-dire les différences de représentations, lui sont - souvent- inhérents, cet article a pour but d'approfondir la compréhension de son processus de (re)production. Autrement dit, plutôt qu'au " quoi ", c'est-à-dire aux caractéristiques de la $\mathrm{CC}$, qui ont fait l'objet comme nous le verrons de nombreux travaux, c'est davantage au «comment» de ces dynamiques sur lequel les développements ci-dessous se focaliseront et entendent contribuer.

Dans ce projet, cet article de synthèse propose une conceptualisation théorique de ce «comment» à l'interface des notions de pratique, de communication et de compétence collective. Nous verrons que ces trois

1 Nicolas Arnaud est professeur associé à Audencia Business School dans le département Management. Il occupe le poste de directeur adjoint du programme Grande École. Ses recherches portent sur la compétence collective, les relations inter-organisationnelles et les nouvelles pratiques de management. Il est l'auteur de nombreux écrits scientifiques et autres articles de presse, tant en France qu'à l'international; nicolasarnaud@ audencia.com 
concepts renvoient à des littératures dont la complémentarité évidente cache, en réalité, un trop faible entrecroisement. Afin de faire avancer les connaissances et les pratiques des entreprises, cette situation rend par conséquent nécessaire un travail d'enrichissement visant à approfondir la connaissance sur les dynamiques de production, de soutien et de transformation de la CC.

En s'appuyant sur la notion de compétence collective telle que théorisée et étudiée par des chercheurs principalement français et québécois, cet article prolonge et approfondit l'étude, à l'aide d'une part des travaux internationaux en communication organisationnelle et d'autre part, de ceux issus d'une approche pratique de l'organisation faisant l'objet d'une forte mobilisation ces dernières années, toujours dans les publications internationales (Cooren et al. 2011, Jarzabkowski et Spee 2009). Trois objectifs sont poursuivis : i) proposer une conceptualisation théorique qui permette d'étudier - avec un haut niveau de granularité- les processus de construction, de transformation et de soutien de la CC et donc de ses caractéristiques (c'est-à-dire des éléments discursif, symbolique et matériel propres aux collectifs); ii) utiliser cette perspective théorique pour proposer de nouvelles pistes de recherche intégrant une dimension plus internationale ; iii) et ainsi participer à « l'exportation » de la notion de CC dans les débats internationaux.

Dans une première section, les enseignements significatifs de ces trois perspectives sont présentés et articulés de manière à mettre en évidence leurs complémentarités. En s'appuyant sur ces fondements, la seconde section propose et discute un programme de recherche favorisant de futurs travaux visant à mieux comprendre les dynamiques de la CC. Enfin, la conclusion propose une définition intégrative et multi-niveaux de la $\mathrm{CC}$, identifie des pistes concrètes de recherches futures et discute des implications méthodologiques.

\section{Compétence collective, communication organisationnelle et pratiques}

Après avoir établi la dimension principalement francophone des travaux sur la CC, et montré que ces derniers se sont davantage intéressés aux productions stabilisées plutôt qu'aux mécanismes à l’ouvre dans sa dynamique de production (le «comment») ${ }^{2}$, nous introduisons les travaux en communication organisationnelle et de la pratique. Nous montrons également en quoi ces deux perspectives peuvent être enrichies tant par les travaux sur la CC que par le programme de recherche développé dans la seconde partie de cet article.

\footnotetext{
2 Nous devons reconnaitre ici l'existence de travaux des années 1990 sur les organisations apprenantes. En effet, Argyris et Schon (2002) ou encore Midler et al. (1997) notamment, ont fait référence au terme de compétence collective et, principalement pour les premiers, évoqué sa dynamique de production. Nous maintenons néanmoins que ces travaux n’ont pas davantage traité la nature communicationnelle de la $\mathrm{CC}$ que les travaux mentionnés en GRH. Au final, la CC y est traitée de manière marginale. En effet, la CC n'est pas leur préoccupation centrale mais plutôt une forme de conclusion de leurs études (sur l'apprentissage organisationnel d'un côté et le mode projet de l'autre). De plus, ces travaux ne sont que très (très) peu cités dans les travaux de GRH sur la CC, or, cet article s'adresse justement à ces derniers.
} 
La compétence collective, une notion essentiellement francophone...

Dans la littérature anglophone, force est de constater que les articles portant explicitement sur le terme de «collective competence» sont rares en nombre et diffus dans le temps. Cette notion a été initialement étudiée dans le cadre d'articles portant sur le portefeuille de compétences des firmes : individuelle, collective et organisationnelle (Burgoyne 1989, Nordhaug et Grenhaug 1994). Au sujet de la CC, Burgoyne (1989:60) précisait : « High levels of individual member competence do not guarantee group or organisational competence and effectiveness, and indeed if high levels of competence are associated with large egos, this may be a barrier to effective collaboration. (...) the challenge of finding ways to develop collective competence will still remain». Nordhaug et Grenhaug (1994 : 94) introduisaient alors l'importance de la dynamique d'interaction comme modalité de production de la CC: "The way in which people interact can contribute to producing new, collective competences». Dix années plus tard, l'objectif de Boreham (2004), en militant pour une perspective collective de la compétence, était davantage dans une logique de dénonciation de l'hyper individualisation des performances des salariés. Enfin, plus récemment, Arnaud et Mills (2012) se sont eux intéressés à la CC à l'interface des relations interorganisationnelles. Trois raisons peuvent être évoquées pour comprendre la faible existence de la notion de collective skill ou competence dans la littérature internationale. Premièrement, ces articles de langue anglaise semblent avoir préféré d'autres concepts pour étudier l'action collective3, concepts d'ailleurs souvent étudiés dans des situations variées (situations extraordinaires voire extrêmes, non longitudinales, non temporalisées et non contextualisées dans le système organisationnel, etc.), sans qu'aucune ne puisse être considérée comme synonyme de la CC.Également, le prisme individualisant des pratiques managériales anglo-saxonnes ne plaide a priori pas pour une perspective collective de la compétence (Boreham 2004). Deuxièmement, ces travaux privilégient davantage la performance économique ("l'output») à l'étude de la performance en train de se faire, c'est-à-dire à l'action (Defélix, et al. 2014). Troisièmement, les travaux francophones comme internationaux, ne se sont pas équipés théoriquement (voire méthodologiquement) de manière adéquate pour se saisir de la dynamique de construction de la CC, en situation, et dans une dynamique temporelle.

En France, depuis la publication des premiers articles sur le sujet (Dubois et Retour 1999, Wittorski 2000), la notion de compétence collective fait l'objet d'un intérêt réel tant dans les travaux de consultants (Le Boterf 2008) que dans

3 La diversité des notions est en effet riche : « teamworking » (Procter et Burridge 2008, pour ne citer qu'eux), « collective action» (Quinn et Worline 2008), « community of practice » (Brown et Duguid 2002), « collective identity " (Hardy, et al. 2005), " collective mind » (Weick et Roberts 1993) souvent traduit par la notion d'intelligence collective, ou encore « collective capability » (Orlikowski 2002). Une discussion minutieuse de ces concepts dépassant largement le cadre de cet article, le lecteur intéressé pourra lire par ailleurs la revue de littérature in Defelix et al. (2014) sur la relation à la performance. 
la littérature académique en sociologie du travail (Zarifian 2009), économie (Biencourt et Jolivet 2000), ergonomie (Wittorski 1997), linguistique (Boutet 2001) et communication (Grosjean et Lacoste 1999), et particulièrement en gestion des ressources humaines depuis une quinzaine d'années (Bataille 2001, Defélix, et al. 2014, Retour et Krohmer 2006). Le point de départ est le constat que le développement de la logique et des outils de gestion des compétences (individuelles) dans les entreprises depuis les années 1990 s'est largement traduit par une individualisation de la relation d'emploi (Cavestro, et al. 2007b : 502), alors même que l'interdépendance entre les personnes et les équipes est devenue, plus qu'hier, une condition de performance et de pérennité des organisations, révélant au passage la dimension stratégique de la compétence collective (Retour 2005). La compétence collective doit en effet apporter un surplus de performance pour l'organisation (dans une considération élargie d'efficience, d'adaptabilité, de flexibilité, de sécurité, d'innovation ou encore de bien-être). La compétence collective a fait l'objet de plusieurs définitions (cf. tableau 1 ci-dessous) mettant en avant la capacité d'un groupe à résoudre une situation "qui ne pourrait être assumée par chacun de ses membres seuls» (Bataille 2001 : 67). Cette littérature s'intéresse tant aux caractéristiques de la CC (c'est-à-dire au «stock» de connaissances au sens de Berger et Luckman (1966)) ainsi qu'aux facteurs favorisant son émergence, mais moins à son processus de construction et de transformation, alors même qu'ils s'accordent à reconnaitre la place constitutive des interactions et de la communication (cf. dernière ligne du tableau 1 page 221).

Les travaux sur la compétence collective invitent à mettre plus particulièrement en avant deux dimensions : (1) son caractère situé, invitant à regarder de près les pratiques des acteurs qui la portent, tout autant que celles de ceux qui l'influencent ; (2) sa dynamique et son caractère interactionnel comme conditions de production, de transformation et de soutien de l'ensemble des éléments de la compétence collective. En effet, jusqu'à aujourd'hui, la littérature sur la CC s'accorde largement sur l'importance d'un tel processus (cf. dernière ligne du tableau 1), mais rares sont ceux ayant entamé une telle entreprise, laquelle nécessite de théoriser tout autant que de problématiser davantage cette dimension communicationnelle et les pratiques in situ susceptibles de la réaliser.

La compétence collective est ainsi représentée comme un ensemble de caractéristiques et de ressources, fruit des «coordinations créatrices» (Hatchuel $1994: 109)$ et le "résultat d'un processus d'apprentissage " (Cavestro, et al. 2007a : 20) dans lequel vont puiser les acteurs des collectifs mobilisés pour agir individuellement et/ou collectivement. Cette dimension créatrice et constitutive a bien été identifiée, mais reste peu connue. Ainsi, Amherdt et al. (2000 : 99) considèrent que "c'est la création de règles et d'objectifs communs partagés et légitimes qui fonde l'action collective, qui permet la coopération entre acteurs et qui aboutit à la création d'une compétence collective ». Parallèlement, 
Koenig (1994 : 297) regarde l'apprentissage organisationnel comme un " phénomène collectif d'acquisition et d'élaboration de compétences, qui plus ou moins profondément, plus ou moins durablement, modifie la gestion des situations et les situations elles-mêmes ». L'enjeu est donc bien ici d'étudier les mécanismes de construction, de stabilisation et d'évolution de ces cadres collectifs qui permettent la coordination et l'action. Dès lors, la prise en compte de la dimension structurante des dynamiques conversationnelles des acteurs et de leurs pratiques constitue une porte d'entrée tant théorique qu'empirique pertinente expliquant ces constructions. Nous posons que la création de règles, de représentations partagées, de confiance, de compétences, etc. dépend autant de l'implication des individus dans les dynamiques communicationnelles en situation de travail, que de la capacité de l'organisation et des managers à les promouvoir et les soutenir (par des outils, des réunions, des technologies, des pratiques managériales, des politiques de GRH, etc.). Tableau page suivante.

Les deux sous-sections suivantes ont pour but de présenter les perspectives organisationnelles et pratiques de l'organisation, et d'en démontrer la pertinence pour la compréhension des mécanismes à l'œuvre dans les processus de production, de soutien et de transformation de la CC.

\section{... invitant à se saisir du rôle constitutif des interactions et des pratiques}

\section{Le tournant pratique}

La perspective de la pratique s'est développée en complémentarité à des approches traditionnelles en sciences de gestion jugées trop ancrées dans une analyse formelle, statique et plutôt réductrice (Geiger 2009) du fonctionnement des entreprises. L'objectif de ce tournant a été de remettre le travail, les processus, et les activités au centre de l'analyse des organisations («bringing work back in» [Barley et Kunda 2001]). Même s'il constitue un champ théorique et méthodologique loin d'être unifié (Corradi, et al. 2010), le tournant pratique (Schatzki, et al. 2001) propose de prendre comme objet d'étude les pratiques contextualisées et les actions quotidiennes des acteurs des organisations. S'intéresser aux pratiques offre ainsi une voie pour comprendre "lorganisation comme elle se réalise ${ }^{4}$ (Miettinen, et al. 2009 : 1310) pour reprendre l'expression de Boden (1990, «the world as it happens»).

Le terme de pratique s'inscrit en réalité dans de longues traditions sociologiques et philosophiques (voir notamment les articles de synthèse de Corradi, et al. 2010, Miettinen, et al. 2009). Comme ces auteurs le font remarquer, 10-15 ans après le tournant pratique, ce champ de recherche ne constitue (toujours) pas une théorie unifiée, mais davantage un «bandwagon » (Corradi, et al. 2010) (un train en marche), même si des voix sélèvent pour

4 « understanding organization as it happens» (Miettinen, et al. 2009 : 1310). 


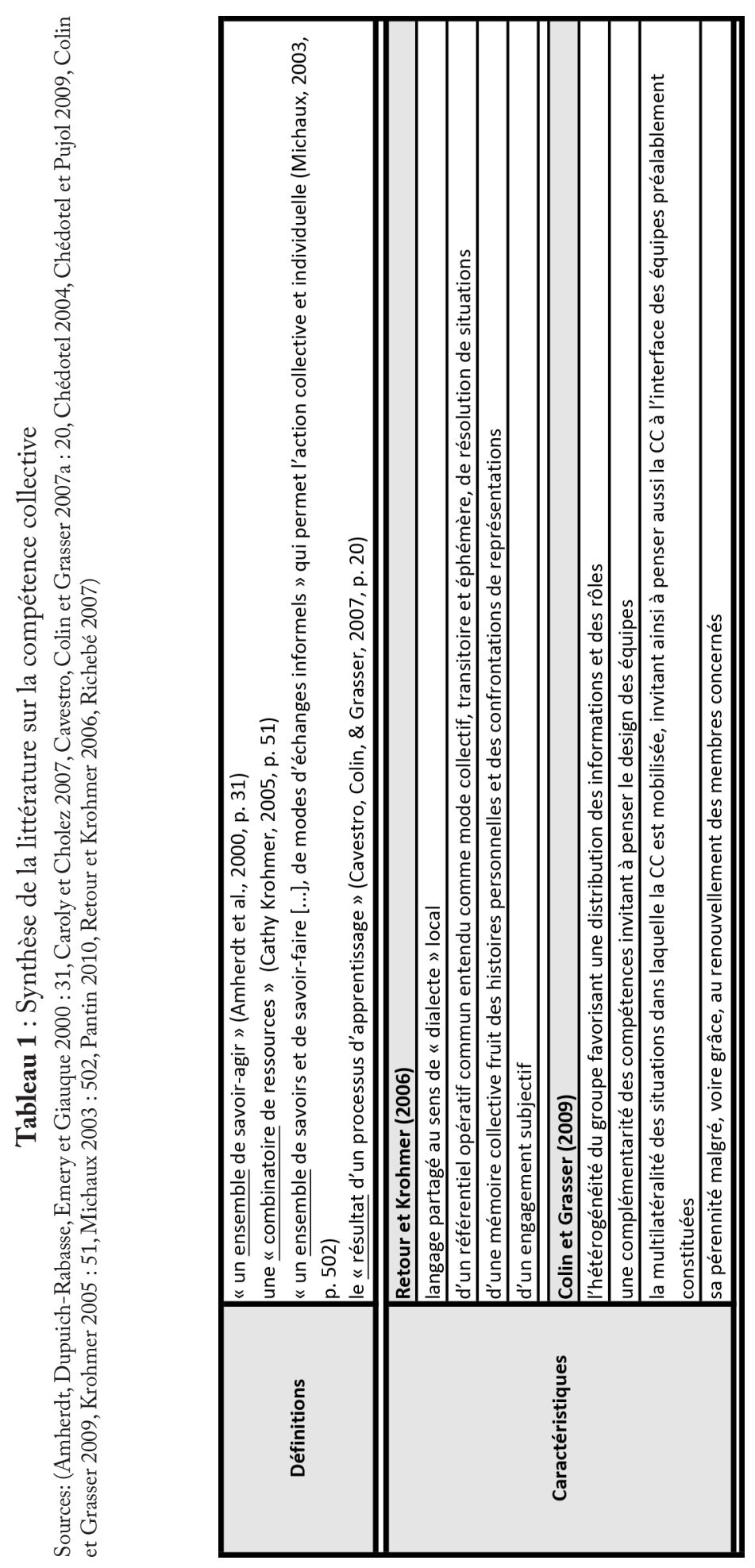




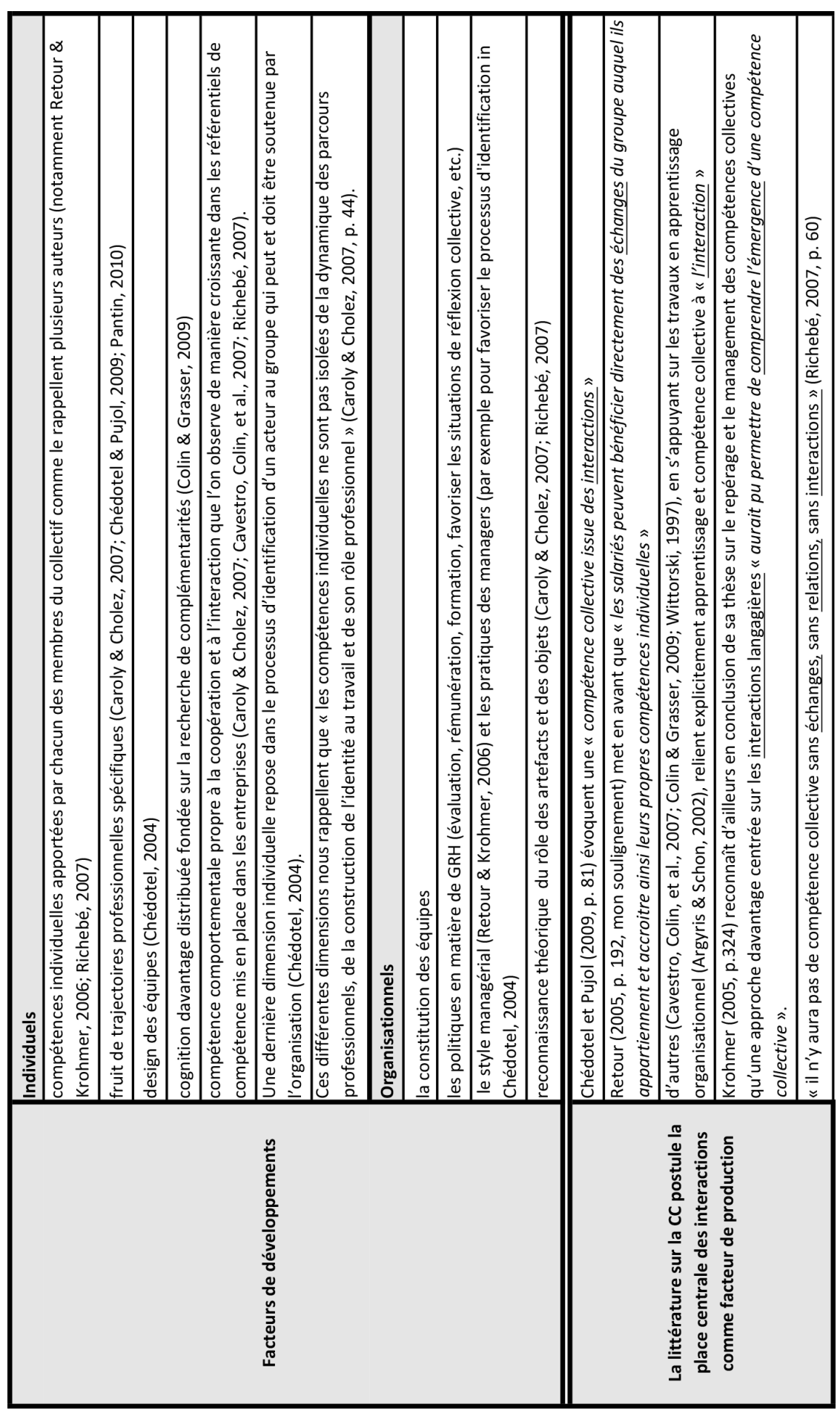


défendre l'idée qu'un certain éclectisme théorique et méthodologique est justifié par le caractère multidimensionnel de la pratique. Corradi et al. (2010 : 267) proposent toutefois la définition suivante de ce courant (re)naissant : " central to the practice perspective is the acknowledgment of the social, historical and structural contexts in which knowledge is manufactured. Practice allow researchers to investigate empirically how contextual element shape knowledge and how competence is built around a contingent logic of action ». Plus largement, le tournant pratique entend approfondir le passage d'une vision individuelle et cognitive de l'apprentissage à une vision située et sociale (et par conséquent collective) (Miettinen, et al. 2009), tel que Brown et Duguid (2001) ont pu l'initier. Le positionnement est clair : puisqu'il est difficile d'analyser les processus cognitifs individuels expliquant les décisions et les actions d'un acteur (d'autant plus que le travail est incontestablement plus interdépendant et collectif qu'hier), les tenants de la pratique militent pour une analyse socialement située de l'activité et une perspective émergente de l'organisation, «enactée» par les pratiques interdépendantes des acteurs.

La perspective de la pratique de la stratégie (PPS) constitue parmi les courants de recherche issus de la pratique, l'un des plus avancés. Son développement en un champ de recherches spécifique s'explique parl'insatisfaction d'un ensemble de chercheurs envers les recherches traditionnelles en stratégie (Jarzabkowski, et al. 2007, Whittington 2006). Dans le sillage des travaux de Whittington (2006), les tenants de ce courant définissent le périmètre de leurs recherches à l'interface des "practitioners (those people who do the work of strategy); practices (the social, symbolic and material tools through which strategy work is done); and praxis (the flow of activity in which strategy is accomplished) " (Jarzabkowski et Spee 2009: 73). D’après Jarzabkowski (2005: 9-10), les pratiques sont constituées de pratiques administratives et discursives (les discours, les conversations ou encore les outils en tant que porteur d'une philosophie gestionnaire), et d'autres renvoyant à ce qu'elle nomme des épisodes (réunions, séminaires, etc.). Le terme de praxis, généralement traduit en français par la pratique (au singulier), est ici entendu comme un corps constitué de savoirs, le plus souvent définis dans un «ici et maintenant» autre que celui de réalisation des pratiques par les praticiens (les consultants, le métier, les prescriptions, etc.). Il renvoie donc à l'idée de spécialité professionnelle.

$\mathrm{Au}$ final, force est de constater que ce tournant pratique, particulièrement exploité en management stratégique n'a, à ce jour, pas (encore ?) été pris, malgré les quelques travaux récents sur les pratiques de recrutement (Llewellyn et Spence 2009) ou de GRH (Vickers et Fox 2010), ou encore en leadership (Carroll, et al. 2008), explicitement ancrés dans cette perspective. Or, cette dernière peut contribuer à des études sur les mécanismes toujours situés et donc ancrés dans les micro-pratiques de constitution des déterminants de la CC comme certains l'y invitent (Minbaeva, et al. 2009). Également, jusqu’à 
récemment, ces travaux ont peu questionné le rôle de la matérialité et des outils de gestion dans les processus organisants (Dameron, et al. 2015).

\section{Le tournant linguistique}

Parallèlement à ce tournant pratique, les sciences de l'organisation se sont également engagées dans une perspective linguistique (Czarniawska-Joerges 1998), discursive (Alvesson et Karreman 2000) et communicationnelle (Cooren, Kuhn, Cornelissen et Clark 2011). Si certains pouvaient regretter, il y a encore une dizaine d'années, la faible importance accordée aux activités de parole dans les recherches en management (Woodilla 1998), la situation n'est plus la même. Ainsi, la parole au travail (Drew et Heritage 1992) fait l'objet aujourd'hui une littérature abondante au sein d'une grande variété de disciplines (sociolinguistique, psychosociologie des relations humaines, interaction machine-humain, ethnographie du langage, etc.). De l'analyse conversationnelle d'une unique réunion (Gephart 1978) à la mise en lumière de la compétence discursive des middle managers (Rouleau et Balogun 2011), en passant par l'analyse des propos d'un ensemble d'interactions enregistrées (Boje 1991), un large panel de méthodologies et de situations a été utilisé/ étudié. Cette évolution a permis la reconnaissance progressive de la fonction constitutive de l'activité de parole («the business of talk», Boden 1994) ainsi que de la dimension organisante/constitutive de la communication sous toutes ses formes (Cooren, et al. 2011).

Dans un exercice de synthèse de ces travaux, Fauré et Arnaud (2014) identifient deux perspectives. La première, d'origine française, ancrée dans l'étude de la transformation du travail et des organisations, étudie l'activité de parole comme un outil de travail (Detchessahar 2003). La seconde, davantage internationale, trouve son point de départ dans le tournant linguistique (Alvesson et Karreman 2000) qu'ont connu les sciences sociales, d'ailleurs en parallèle du tournant de la pratique (des exemples significatifs sont notamment les travaux de Cooren 2000, Taylor 1993).

Quels que soient les niveaux d'analyse ou les ancrages théoriques, la communication y est perçue comme un processus complexe et incertain de construction de sens impliquant une participation active des membres (Detchessahar 2003,Putnam et Pacanowsky 1983) et constitutif de l'organising (Cooren 2000, Cooren, et al. 2011). Ces auteurs ne s’intéressent pas tant à la manière dont les individus échangent des informations et des connaissances, mais plutôt à la façon dont collectivement, et de manière interactionnelle, ils construisent (ou ont construit) des connaissances pour agir ensemble (Arnaud 2011, Cooren, et al. 2006). Fortement inspirée des travaux de Giddens (1984) sur la dualité du structurel, cette perspective considère que les connaissances constituent à la fois le prérequis de leurs interactions, et un résultat devenant 
l'une des bases mêmes de leur collaboration et qu'elles sont amenées à évoluer en fonction des interactions quotidiennes des acteurs, des problèmes qu'ils rencontrent, mais aussi en fonction de l'arrivée de nouveaux participants, humains ou non (cf. ci-dessous la figure 1). Le caractère (ac)cumulatif de cette perspective fait directement écho au processus de structuration chez Giddens (1984), aussi appelé laminage chez Boden (1994) ou sédimentation chez Berger et Luckman (1966).

Si ces travaux ont significativement contribué au renouveau des approches interpretativiste/communicationnelle de l'organisation et de l'entreprise (Fauré et Arnaud 2014), le type d'études empiriques réalisé a pour l'heure peu permis d'y intégrer le rôle du management. En effet, les analyses empiriques se sont focalisées sur l'analyse de situations extraordinaires (Cooren et Fairhurst 2004) ou de réunions de comité de direction (Cooren 2004), dans la droite ligne des recherches précédentes (notamment Boden 1994, Weick et Roberts 1993). De plus, ces situations concernent des acteurs qui souvent ne se connaissent pas et n'auront vraisemblablement pas à interagir ensemble dans un futur proche et déterminé (voir par exemple la conversation entre un client et un agent immobilier in Cooren, 2004). Dès lors, les analyses ne s'inscrivent pas dans une perspective temporelle et, au moins partiellement, stabilisée impliquant une dynamique d'interactions entre acteurs qui est celle des situations d'entreprises relevant d'un périmètre d'action managériale. Il n'est donc finalement pas surprenant que ce prisme de l'action managériale n'intéresse pas ou peu les travaux en communication organisationnelle (Arnaud et Mills 2012). Or, justement, sur ce point, les sciences de gestion ont toute leur place. Également, comme nous le verrons ultérieurement, à l'instar de la perspective de la pratique, les acteurs au cœur des conversations manquent singulièrement d'épaisseur sociale et la matérialité n'a que très récemment commencé à être prise en compte (Cooren et al. 2012).

Au final, ces travaux ont démontré que les pratiques communicationnelles des acteurs produisaient, sous certaines conditions, des ressources au service de la compétence du collectif. Parallèlement, une autre littérature démontre l'existence et la forme de pratiques managériales dont l'une des finalités est aussi de produire de la compétence à différents niveaux de l'organisation (équipes opérationnelles, collèges de pairs, comités de direction). Finalement, au carrefour de ces deux approches, il est possible de s'interroger i) sur les formes de communication, et ii) ce qui se passe dans la communication qui produit de la compétence (ou pas) pour iii) finalement identifier les dimensions managériales et organisationnelles qui influencent sa production.

\section{Vers une perspective communicationnelle et pratique de la construction de la compétence collective}

Dans un article récent, illustratif des travaux sur la $\mathrm{CC}$, Chédotel et Pujol (2012 : 5-6) précisent ainsi que la compétence collective repose sur une 
confrontation des représentations devant aboutir à une décision collective adaptée à la situation. Ces deux auteurs s'appuient sur une enquête qualitative reposant sur 55 entretiens auprès de dirigeants, d'administrateurs et de salariés de SCOP pour montrer l'existence (ou non) d'une compétence collective, c'est-à-dire ici le degré de collectif impliqué directement dans la prise de décisions stratégiques de ces organisations. Sans remettre naturellement en cause la qualité et la pertinence de cet article, et tout en reconnaissant qu'il est difficile d'aborder plusieurs dimensions dans un même article, une telle conception de la compétence collective permet d'introduire les fondements du programme de recherche proposé dans cet article. Premièrement, considérer la compétence collective comme l'expression d'une décision collective ponctuelle ne permet pas de prendre en compte ses caractéristiques dynamiques et temporelles, et le fait que cette compétence collective soit en (re)construction permanente justement à travers des processus, voire des luttes (Vaara 2010), d'essence discursive et narrative. En d'autres termes, la compétence collective, que l'on s'y intéresse au niveau d'un comité de direction ou d'une équipe opérationnelle, nécessite de mettre au jour sa généalogie, c'est-à-dire le processus de construction (non automatique) de ses ressources sociales objectivées dans le stock de connaissances de la CC. Deuxièmement, et dans le prolongement du premier argument, la compétence collective, parce qu'elle invite à comprendre et mettre en lumière le caractère situé et singulier de la situation ou du problème à résoudre, ne peut faire l'impasse sur la structure organisationnelle et managériale (système d'évaluation, organisation du travail, politique de recrutement, structure de la gouvernance, pratiques du management de proximité, etc.) toujours spécifique et influençant son émergence et son action. Troisièmement, vouloir comprendre ce qui se joue dans la compétence collective, c'est également (et peut-être paradoxalement) vouloir éclairer ce qui se joue d'individuel (c'est-à-dire au niveau des praticiens, des acteurs) dans le travail.

La compétence collective est par conséquent réalisée et mobilisée par des acteurs encastrés dans des univers narratifs et discursifs, organisationnels et hiérarchiques, ainsi qu'identitaires spécifiques. Le programme de recherche proposé ici invite : 1) à approfondir la question de l'acteur dans sa relation à ses compétences et ses pratiques ; 2) à creuser la dynamique de co-construction de la compétence collective c'est-à-dire la relation individu/collectif; et 3) à comprendre comment les pratiques managériales et plus largement le cadre organisationnel influencent les dynamiques précédentes.

Ainsi, nous posons que comprendre la dynamique de (re)construction de la compétence collective nécessite de s'intéresser aux acteurs, aux luttes narratives (les conversations participant à la (re)définition des pratiques, de l'activité, voire de l'organisation) et au système organisationnel et managérial constitué des politiques managériales et des règles/procédures ainsi que des 
pratiques des managers (supérieurs hiérarchiques). Ces trois dimensions sont en interdépendance les unes par rapport aux autres et participent directement au développement (ou non) de la compétence collective. Chacun de ces axes constitue des pistes en soi de prolongements et d'approfondissements de la CC ; combinés, articulés et mis en perspective avec les développements précédents sur les tournants pratique et communicationnel, ils peuvent constituer l'architecture d'un programme de recherche plus vaste.

Cette perspective traduit la dimension dynamique et processuelle de notre proposition, dans une perspective d'apprentissage collectif à travers la communication et les pratiques (Corradi, et al. 2010, Nicolini, et al. 2003). Parce que les acteurs construisent dans l'interaction des connaissances pour agir ensemble, ces dernières constituent tant le pré requis de leurs interactions que leurs résultats, devenant les bases mêmes de leur collaboration à venir. Ces connaissances peuvent prendre la forme de langage opératoire commun, de décisions collectives, d'histoires partagées, de marqueurs de confiance, des modes d'utilisation des outils et de la matérialité, etc. et participent à la construction d'un cadre partagé pour l'action. Leur utilisation en pratiques constitue des signes de la CC. Dans ces processus interactionnels, la construction du sens de la situation, et donc de l'action, ne va pas de soi. Elle implique que chacun mette en mots et accepte de confronter ses représentations à autrui. Également, dans ce processus les pratiques managériales et le système organisationnel participent au cadrage des situations rencontrées, voire orientent le comportement du groupe et des individus.

Les développements ci-dessous présentent les implications de ces trois dimensions. A des fins analytiques, ces développements s'intéressent aux acteurs en mettant en discussion deux conceptions sociologiques distinctes de l'identité personnelle ainsi qu'en en montrant l'actualité dans les débats académiques actuels. Dans un second temps, la notion de lutte narrative comme condition de construction et d'amendement des représentations individuelles et collectives de l'organisation et du travail est exposée. Enfin, cette discussion théorique se terminera en abordant le rôle du cadre organisationnel et managérial en tant quélément structuré et structurant pour le développement d'une compétence collective à différents niveaux de l'organisation.

\section{Acteurs}

Les acteurs analysés dans les études se revendiquant tant de la communication organisationnelle que de la perspective de la pratique manquent paradoxalement souvent d'épaisseur sociale. En effet, ces travaux ne s'intéressent pas ou peu aux acteurs. Or, comprendre leurs trajectoires personnelles, leurs expériences passées, leurs compétences développées (Caroly et Cholez 2007), leur compréhension du système d'action concret dans lequel ils évoluent, ou encore leurs traits de personnalité peut permettre de mieux 
saisir l'origine de leurs pratiques, et de les interroger sur le sens qu'ils donnent à leurs actions ainsi qu'aux situations qu'ils rencontrent. Les différences entre individus impactent la perception qu'ont les acteurs de leur travail, de leur organisation, et donc de leur périmètre et de leur forme d'action.

Dans le prolongement de l'analyse conversationnelle telle que développée par Sacks et al. (1974), les tenants de la communication organisationnelle entendent étudier l'interaction en tant que processus complexe de coordination des actions d'une part, et en tant qu'accomplissement pratique d'autre part. Dans cet objectif, ils ne s'intéressent pas aux acteurs pour autre chose que ce qu'ils disent.

Lobjet de l'analyse de conversation est le discours dans l'interaction, le discours en tant qu'il a été produit conjointement par deux ou plusieurs participants. L'analyse de conversation part du fait que l'interaction verbale procède de façon ordonnée et quelle possède, à ce titre, une structure complexe organisée séquentiellement au moyen du système des tours de parole. Les participants à une interaction peuvent utiliser comme ressource fondamentale l'existence de cette structure pour organiser et accomplir de façon située leurs interactions. (de Fornel et Léon 2000 : 144)

L'analyse de conversation, parce qu'elle se limite volontairement à ce que disent les acteurs, permet d'éviter de faire l'hypothèse que ces derniers ont des représentations ou des cognitions (Latour 2002) préalables à toute conversation. Autrement dit, on ne s'intéresse pas ici aux expériences antérieures des participants aux conversations, ni à leur travail identitaire.

Il est possible d'observer une même réserve au sein des travaux issus de la perspective de la pratique, et ce, malgré un objectif théorique annoncé, notamment par Whittington dès 1996 et réaffiché plus récemment (Rouleau 2013). L'invitation de Whittington à prendre en compte dans l'analyse l'intégralité du triptyque "praxis, practice, practitioners " n'a donc pas été systématiquement suivie, particulièrement du point de vue des acteurs. D'où viennent-ils? Quelle est leur trajectoire socioprofessionnelle ? Quelles sont leurs caractéristiques comportementales? Et en quoi ces dernières influencent-elles leurs pratiques? Et inversement? En effet, les travaux relevant du strategizing, lorsqu'ils portent sur les «practitioners» (notamment Laine et Vaara 2007) consistent essentiellement à identifier qui est stratège et qui ne l'est pas, et ce qu'il fait en pratique. C'est en cela qu'il est possible de considérer qu'il manque une certaine épaisseur sociale aux travaux issus de la pratique portant sur les praticiens. Lidée que tout acteur apporte au travail son vécu, sa trajectoire, ses préférences comportementales, etc. influence sa représentation des situations, sa capacité à leur donner du sens et donc à influer sur la représentation d'autrui, et par extension ses pratiques, devient alors centrale. Ce type de connaissance augmente en effet considérablement notre compréhension des situations tout en permettant d'imaginer des politiques $\mathrm{RH}$ adaptées (sélection, formation, carrière, rémunération). Dans cette 
quête, des outils méthodologiques existent et des articles en entrepreneuriat institutionnel notamment (Downing 2005), mais aussi en management d'équipes (Chédotel 2004), ont commencé à s'intéresser à ce sujet. Comprendre la trajectoire socioprofessionnelle des participants à l'activité constitue un élément important de la bonne compréhension des situations sociales. Les recherches proposant une articulation entre acteurs et pratiques doivent ainsi être encouragées en s'interrogeant sur les grands fondements de l'identité personnelle, dans une conception narrative davantage que sociale (Johnson, et al. 2010).

Étudier ce que sont les personnes amène en effet à prendre quelques précautions afin d'éviter tout déterminisme (notamment sociologique) qui rendrait toute analyse de l'activité non pertinente au motif que tout serait à voir et à comprendre en dehors de cette dernière, à savoir dans les grands déterminismes sociologiques tels que l'habitus ou la socialisation chez Bourdieu. Sur ce point, le débat est toujours d'actualité et rend nécessaire une prise de position. Loin de penser avec Bourdieu $(1980,1986)$ que les pratiques de l'être humain soient entièrement réductibles à ces déterminants, rendant ainsi illusoire toute recherche du sens de la pratique dans la biographie, je penche plus sensiblement pour l'idée de compétence narrative (entendue comme capacité des acteurs à (se) raconter) proposée par Ricœur (1990) pour expliquer la dimension profondément fraiche, émergente et potentiellement créative de l'activité humaine. Très clairement chez Bourdieu, «l'identité pratique» est produite par l'habitus individuel et constitue à ce titre le principe actif de "l'unification des pratiques et représentations" (Bourdieu 1986 : 70). Ricœur, s'il reconnaît le poids des déterminismes sociologiques sur l'identité personnelle et l'action humaine, est plus mesuré et surtout reconnait ouvertement que lêtre humain ne peut être totalement réduit à son être social. Pour reprendre ses termes, une part d'ipséité résiste toujours notamment dans la fidélité à la parole donnée5. Dès lors, l'identité personnelle doit être complétée par ce que Ricœur nomme l'identité narrative. Le récit de vie permet à chacun de se (re)constituer une interprétation de soi (parmi d'autres possibles) et d'autrui, c'est-à-dire de réunir la mêmeté et l'ipséité. Clairement pour Ricœur, la narration constitue «l'unique ressource pouvant permettre d'articuler à la dimension pratique de l'identité personnelle (babitus-mêmeté) cette part d'ipséité singulière qui n'est pas moins constitutive de la personne» (Truc 2005 : 54). Autrement dit, l'identité narrative occupe donc une place médiane entre "deux modèles de permanence dans le temps» (Ricœur 1990: 143), le

\footnotetext{
5 L'ipséité «désigne un autre mode d'identité et un autre modèle temporel, selon Ricœur: « une chose est la persévérance du caractère (notion de Idem ou mêmeté chez Ricœur, proche de la notion d'habitus de Bourdieu); une autre, la persévérance de la fidélité à la parole donnée » car « la parole tenue dit un maintien de soi qui ne se laisse pas inscrire, comme le caractère, dans la dimension du quelque chose en général, mais uniquement dans celle du qui ? " Le caractère est permanence du temps en tant que maintien du même, la parole tenue constitue au contraire, par le maintien de soi, « un défi au temps, un déni de changement ». Par l'ipséité, l'autre demeure en soi-même. » (Truc 2005: 53)
} 
premier renvoyant au caractère/mêmeté (proche de l'habitus) et le second au maintien de soi dans la promesse donnée (ipséité), soit l'homme dans ses liens. Dans l'ipséité, la seule permanence de l'homme devient la fidélité dans ses engagements, c'est-à-dire son engagement dans ses interactions, soit pour ce qui nous intéresse directement, dans les dynamiques de construction de la compétence collective. Les récits servent alors de cadrage et alimentent une identité individuelle et collective en devenir à travers la discussion et la confrontation (que nous appelons plus bas lutte narrative). La pratique n'est par conséquent jamais close et constitue un processus qui n'a jamais de fin, tout comme la construction de son sens (Johnson, et al. 2010). Ce positionnement théorique invite à rechercher cette construction du sens des situations sociales, ou plus exactement de processus sociaux toujours en mouvement, à la fois dans l'entretien biographique type récit de vie et de pratiques, mais également dans une triangulation avec des observations d'activité. Les trajectoires sont importantes, non pas pour sortir de l'analyse de l'activité, mais bien davantage pour s'attaquer aux questions de l'identité parce que, dans cette activité, qui a un caractère irréductible de singularité, se joue en réalité un phénomène de recomposition permanente de ces identités. La mise en place de nouvelles pratiques de management favorisant le collectif a des conséquences au niveau individuel et engendre souvent une redéfinition profonde de la façon dont l'individu pense sa relation vis-à-vis du collectif, et le manager son rôle et ses pratiques. Comprendre ces questions identitaires, c'est se plonger dans les conditions personnelles expliquant l'engagement et la subjectivation des acteurs dans les dimensions collectives du travail.

\section{Luttes discursives}

Loin de l'unique voix de l'organisation (Taylor et Cooren 1997), des auteurs proposent de s'intéresser aux luttes (struggles) discursives se cachant derrière cette unicité (souvent apparente) du discours managérial et/ou organisationnel (Phillips, et al. 2004, Vaara 2010). Ces luttes constituent la matérialisation empirique des confrontations de représentations. Comme le précise Vaara (2010), il est possible d'étudier ces luttes à différents niveaux de l'organisation : micro, dans les conversations et les réunions ; méso, dans les pratiques des managers et leur rôle d'intermédiaires; et macro, dans les grands discours organisationnels des dirigeants. Dans cet objectif, la notion de métaconversation, issue des travaux de l'école de Montréal autour de la dualité conversation-texte développée par Robichaud et al. (2004), constitue un point de départ intéressant. En effet, cette notion est présentée comme un moyen de relier des conceptions opposées de l'organisation considérée à la fois comme "pluralistic and unitary, multivocal and univocal, polyphonic and monophonic, many and one » (p. 618). Cette notion se situe à différents niveaux en ce sens qu'elle transcende la dualité micro/macro qui influence 
en profondeur les interactions humaines. Une métaconversation peut être identifiée à l'interface des activités de paroles et des textes (écrits, matériels et oraux). Pour Fenton et Langley (2011), ces travaux impliquent une cohérence organisationnelle dynamique en s'appuyant sur les interactions de multiple processus. Dans cet objectif, il s'agit notamment de comprendre comment et pourquoi telles ou telles histoires, pratiques, modes d'usage d'un outil, etc. deviennent plus (ou moins) influents ? Comment et pourquoi certaines formules rhétoriques deviennent ou non légitimes pour établir/imposer une stratégie ou un nouveau processus organisationnel ? Il devient alors nécessaire de mettre au jour le processus itératif de construction de la mise en cohérence des discours et des pratiques du top management aux opérateurs en passant par les managers intermédiaires. La réalité de la vie des organisations repose sur la confrontation des représentations des individus lors de situations de travail variées allant de la résolution d'un problème opérationnel (un colis est considéré comme manquant, c'est la seconde fois pour ce client en deux semaines, que doit faire l'équipe d'exploitants ?) à la prise de décision stratégique par un comité de direction (quelle est la meilleure modalité de développement stratégique ?) en passant par des questions d'organisation du travail à gérer par des managers de proximité (quelles sont les modalités organisationnelles adéquates pour résoudre des problèmes de délais d'ouverture de compte ?). Si travailler, c'est confronter des représentations différentes du réel à travers la conversation, alors ces luttes discursives sont au cœur du fonctionnement de nos organisations et de la compétence collective. Ces luttes narratives, lorsqu'elles prennent la forme de conversations, servent de point de contrôle des éléments retenus dans le stock de connaissances de la compétence collective. Leur mise en discussion permet la validation par le groupe et sert alors de ressources pour un temps, pour faciliter la réalisation de l'activité à un niveau individuel et/ou collectif. Toutefois, cette confrontation des représentations à travers la communication intersubjective ne va pas de soi. En effet, parce qu'elle concerne des dimensions d'ordre politique, de compétence tant métier que discursive, et parfois stratégique, la confrontation se situe toujours dans un continuum entre consensus et conflit. La confrontation, pour qu'elle puisse produire une représentation commune et une cohérence des discours, nécessite que les acteurs aient le sentiment que tout a été dit, et qu'aucun n'a tenté de manipuler autrui (Detchessahar 2013). Ce dernier point est important, car il participe à la construction d'une relation de confiance entre acteurs, élément fondateur de la compétence collective.

Il faut toutefois se garder de toute crainte ainsi que de tout enchantement de la lutte discursive. Si elle est constitutive de la vie des organisations et des collectifs de travail et constitue à ce titre un moteur de l'activité et de la compétence collective, les responsables d'organisations ne doivent pas y voir (seulement) une source de tension de l'apparente unicité organisationnelle, mais au contraire bien comme une condition de possibilité. Par conséquent, 
il ne faut pas en avoir peur, il ne faut pas l'éteindre, au contraire, mais bien penser ses conditions de soutien, de cadrage et d'accompagnement et surtout accepter que cela ne soit jamais fini. Clairement, la lutte discursive n'est pas un problème de la vie des organisations. Au contraire, elle constitue un des moteurs de la communication intersubjective et qui, bien loin de nous éloigner de la compétence, en réalité en crée (Arnaud 2011). Il faut en revanche être capable, à titre individuel, et collectif, d'accepter tout ce qui pourra être dit dès lors qu'il n'y a aucun doute sur la volonté des participants à travailler pour le bien du collectif et de l'organisation. Il s'agit alors d'avoir un lieu pour rendre en permanence visible ces conflits de manière à fabriquer des consensus qui sont par nature éphémères et qui seront retravaillés à travers d'autres luttes, ce que A.D. Brown (2006 : 735) qualifie de « constant state of becoming ». Or ce type de lieu ou moment de communication intersubjective ne vont pas de soi, tant nombre de réunions constituent davantage des lieux de transfert d'informations que de mise en discussion du travail (Detchessahar 2013). Ces lieux peuvent par ailleurs se trouver lors de la réalisation de l'activité ellemême à condition qu'elle soit permise/autorisée, mais aussi soutenue par des outils et cadrée par des managers eux-mêmes experts tant du travail que de l'animation de la communication intersubjective. Pour cela, la sensibilité des dirigeants envers ce type de pratiques est importante. Ils doivent les officialiser et les soutenir à travers notamment les pratiques et les outils du management.

\section{Cadre organisationnel}

L'objectif de cette dernière section est d'intégrer dans l'analyse le caractère structuré et structurant du cadre organisationnel dans lequel la compétence collective se réalise. Davantage que des réponses abouties, il s'agit d'ouvrir la réflexion sur la ou les formes que peuvent et doivent prendre le cadre organisationnel et les pratiques des managers (entendus comme supérieurs hiérarchiques des acteurs étudiés de la compétence collective) pour favoriser la compétence collective, tout autant que de discuter l'implication de certaines pratiques couramment observées en entreprises.

Comme expliqué plus haut, la perspective constitutive de la communication défend l'idée que l'organisation est par nature fondamentalement émergente et peu stabilisée. La question de la coordination étudiée à travers le prisme de la conversation s'appuie sur le principe de coorientation (Taylor 2006) entre individus. De manière non exhaustive et complémentaire aux différents éléments rapportés jusqu'ici, trois dimensions sont développées. Ces dernières doivent être entendues comme des éléments structurant le cadre managérial au sein duquel le travail est réalisé.

Premièrement, les pratiques discursives et narratives, et plus spécifiquement le recours instrumental au storytelling par les managers, constituent des forces organiques puissantes de la vie des organisations (Boje 1991, Gabriel 2000) 
et par extension du cadre organisationnel dans lequel se réalise toute action collective. Ces pratiques constituent en effet des mécanismes de production et de partage de savoirs importants (Birch 2000) lorsqu'elles sont utilisés de manière instrumentale (Bray, et al. 2000). Des travaux récents ont par exemple montré que les récits constituaient de véritables outils de stratégies et de management en permettant de promouvoir des objectifs organisationnels (Tyler 2007). Toutefois, en écho à la section précédente, tout récit utilisé de manière instrumentale par le management peut naturellement être remis en cause, voire mis en débat, par les différentes parties prenantes (Boje, et al. 1999, Tyler 2007). Ils se trouvent alors au centre de relations de pouvoir (Boje et Smith 2010, Hardy et Phillips 2004) et par conséquent au cœur des luttes discursives (Vaara 2010) à différents niveaux de l'organisation.

Deuxièmement, un ensemble de travaux français a identifié des coûts liés à la mise en discussion du travail. Les salariés sont parfois réticents à s'impliquer dans les logiques discursives et d'autonomie, car cela leur «coûte» d'un point de vue cognitif, politique, social ainsi qu'en terme de responsabilisation (Detchessahar 2003). Changer l'organisation du travail autour d'une plus grande implication des salariés opérationnels dans les espaces de (re)construction de l'organisation fait naître de nouvelles attentes impliquant de faire évoluer la relation d'emploi. Puisque la nature du travail demandé a évolué, la nature des engagements de l'organisation à leur égard (prime, carrière, sécurité d'emploi, rémunération) doit évoluer (Honoré 2002) au risque de générer de la frustration donnant lieu à un retrait de l'engagement. Cette invitation implique de s'intéresser au travail réel, tel que vécu et réalisé par les salariés. Elle nécessite également d'impliquer davantage les salariés dans la coconstruction des modalités de réalisation et d'évaluation de travail collectif de manière à éviter que celui-ci soit constitué d'objectifs non négociables corrélés avec des moyens imposés.

Un troisième élément repose sur le rôle des objets pour structurer et animer les pratiques (Cooren, Fairhurst et Huët 2012, Dameron, et al. 2015). De nombreuses recherches ont démontré leurs contributions à la dynamique interactionnelle. L'un des problèmes des outils de gestion (systèmes d'information, grille d'évaluation des compétences, etc.), c'est qu'ils sont souvent prisonniers d'une pensée magique (Markus et Benjamin 1997) parce qu'institués par des consultants et des directions d'entreprises ayant intérêt à promouvoir l'innovation managériale (souvent de manière générique) tout en en minimisant le caractère problématique. L'informatisation passe par un processus de rationalisation de l'organisation en partant des réalités concrètes du travail qui sont ensuite analysées et modélisées pour tendre vers une réalité épurée, cohérente et d'une plus grande rationalité. Comme bon nombre de méthodes de changements proposées aux décideurs, l'informatisation repose sur des postulats normatifs de rationalisation et de transparence. Or, justement, problématiser l'effet des outils de l'organisation doit permettre de 
mieux comprendre leurs effets sur le travail et par conséquent remettre les pratiques managériales au cœur du travail réel. La construction du sens en situation de travail implique de mettre en lumière l'influence non seulement des dimensions sociales, mais aussi, et surtout matérielles (outils, tableaux, dispositif de gestion) et virtuelles (TIC) (Bakke et Bean 2006), ce que Mills (2009) nomme "geosocial environment». Selon Bakke et Bean (2006 : 56), il est nécessaire de dépasser les approches cognitives et communicationnelles $\mathrm{du}$ travail et considérer les organisations comme "a corporeal body of embodied-thinking-thinkers, who are acting and interacting in a spaces [sic] that share material arefacts... ». Mieux comprendre les dimensions matérielles et virtuelles du travail est insuffisant. Les nouveaux outils ne font rien par euxmêmes, ils ne changent l'entreprise qu'à la condition que les individus se les approprient et les utilisent en pratique et qu'ils constituent davantage des outils supports à l'interaction que de contrôle des activités (Moisdon 1997).

\section{Conclusion: Définition, pistes de recherches futures et implications méthodologiques}

Cet article propose d'appréhender la CC à l'interface de la communication organisationnelle et de la perspective de la pratique. Il vise ainsi à approfondir le «comment» de la $\mathrm{CC}$. Ce travail de synthèse permet i) de formuler une perspective intégrative et multi-niveaux de la compétence collective; ii) de proposer des pistes de recherches concrètes; iii) d'en esquisser quelques implications méthodologiques.

\section{Une perspective intégrative et multi-niveaux de la CC}

À la lumière des développements précédents, une nouvelle définition de la compétence collective peut être formulée en prenant en compte son processus de développement, ses modalités de soutien et l'hétérogénéité des acteurs (humains et non-humains) concernés. Ainsi, une compétence collective peut s'entendre comme l'ensemble des ressources d'un collectif permettant l'action collective et individuelle en situation. Tout collectif est composé de ressources hétérogènes à la fois humaines, symboliques et matérielles, sur lesquelles il est possible d'agir, et qui sont mises en relation à travers l'activité communicationnelle des acteurs et de l'organisation (Robichaud 2006). Un cadre collectif d'action est alors établi. Ce cadre, tout comme les connaissances qui le constituent, évolue au gré des interactions successives des participants et de l'incorporation de nouveaux actants (recrues, mise en place de nouveaux outils, etc.). De nouvelles connaissances sont créées et incorporées au stock de la compétence collective. Toutefois, les connaissances antérieures ne disparaissent jamais totalement. Elles sont, au contraire, amenées à être rediscutées et adaptées, par les acteurs, selon les nouvelles caractéristiques des situations rencontrées. 
Si les travaux sur la CC ont affirmé, ici et là, la dimension structurante des interactions (Dubois et Retour 1999) ou encore la place centrale des confrontations de représentations (Chédotel et Pujol 2012), nous considérons que, faute de grilles théoriques et de manière concomitante de design de recherche adaptée, ces derniers n'ont pas réussi à expliquer et à rendre pleinement compte des dynamiques puissantes - de nature communicationnelle- de construction de la CC à l'œuvre. Ainsi, la proposition défendue dans cet article se singularise par : i) son focus sur la dynamique et le processus pour comprendre les caractéristiques et les productions de la CC (son stock); ii) la prise en compte de la matérialité des situations de travail comme participant à la dynamique collective; iii) la relation acteurs/pratiques à travers des travaux sur l'identité comme entrée pertinente pour comprendre l'engagement dans les dynamiques collectives et le management en pratique ; iv) son caractère multiniveaux.

Plus spécifiquement sur ce dernier point, l'un des intérêts de l'approche proposée est qu'elle peut s'appliquer à différents niveaux de l'organisation et en même temps à toute l'organisation (équipes opérationnelles, interéquipes, équipes interorganisationnelles, collèges de pairs (i.e.,managers intermédiaires) voire comités de direction). Appliquée aux hauts dirigeants, il est par exemple envisageable d'étudier les conditions d'existence d'une compétence collective se construisant par la communication intersubjective et s'appuyant sur les identités personnelles et collectives particulières. Appliquée aux managers de proximité, il devient alors possible d'enquêter sur la construction d'une compétence collective entre pairs et/ou au sein de leurs équipes. Appliquée à plusieurs niveaux d'une organisation, il devient possible d'observer la manière dont les luttes narratives constituent un moteur ou un empêcheur de cette compétence. Dans cette quête, comprendre avec précision le caractère structurant du système organisationnel (c'est-à-dire l'organisation du travail, le système RH, la structure de la gouvernance) et des pratiques managériales est central.

La perspective proposée dans cet article invite également les travaux en communication organisationnelle à intégrer davantage le prisme managérial autour des possibilités de cadrage et de soutien des processus communicationnels à l'œuvre dans les situations de travail. Aux perspectives communicationnelle et pratique, ce programme de recherche propose des clés pour une plus grande prise en compte de l'épaisseur sociale des acteurs ainsi qu'une intégration du rôle constitutif de la matérialité dans les dynamiques de production de la CC.

\section{Des pistes pour de futures recherches}

Trois axes de recherche ont été identifiés et discutés: mieux comprendre le lien acteurs/pratiques; recourir davantage à l'analyse multiniveaux permettant 
d'accéder aux luttes discursives au sein des organisations; et identifier, définir et évaluer l'influence des dimensions organisationnelles et des pratiques managériales sur la CC. Ce programme de recherche autour des enjeux de production, de transformation et de soutien de la $\mathrm{CC}$ dans une double perspective pratique et communicationnelle pourra être abordé dans un avenir proche sous l'angle d'une ou plusieurs des quelques questions de recherche ci-dessous formulées ${ }^{6}$ :

- Quelles pratiques managériales soutiennent les dynamiques conversationnelles? Quels pratiques et/ou systèmes RH sont-ils à même de mieux les soutenir?

- Quelles places, et quelles spécificités, pour les compétences collectives à l'interface des équipes voire des organisations?

- La compétence collective au service de quel(s) objectif(s) ? Innovation ? Bien-être au travail ? Performance économique ? Qualité ? ...

- Comment se matérialisent les luttes discursives ? Comment construire la cohérence entre les différents niveaux d'une organisation ? Quelle(s) place(s) et quelle valeur pour les conflits dans les entreprises et le management?

- Quel rôle joue l'identité des praticiens sur leurs pratiques et/ou leurs conversations ? Et comment se développe alors la légitimité d'une pratique plutôt qu'une autre ? Quelle place pour les émotions des praticiens dans la compréhension de leur travail identitaire et de leurs pratiques?

- Aujourd'hui tout est numérisé et communiqué toujours plus vite et plus loin, mais peut-on dire qu'un vent nouveau souffle sur les pratiques de management et l'action collective ? Quelle est la place de l'arbitrage humain dans un monde de plus en plus peuplé d'artefacts?

\section{Des conséquences méthodologiques}

Enfin, ce projet de recherche implique, de manière non exhaustive, deux conséquences méthodologiques pour étudier le «comment» de la construction de la CC. Tout d'abord, en écho à la proposition de Whittington (2011) invitant les chercheurs mobilisant la perspective de la pratique à se "discipliner » au maximum tant d'un point de vue théorique que méthodologique, il apparaît en effet nécessaire de structurer encore davantage les recherches relevant de la perspective de la pratique.Il est en effet possible de gagner en profondeur et en pertinence en ayant plus systématiquement recours à l'analyse longitudinale ainsi qu'à une collecte de données à différents niveaux de l'organisation.

Également, la mobilisation d'outils et/ou d'approches théoriques spécifiques permettant de mieux comprendre la relation acteurs / pratiques constitue

$6 \mathrm{Au}$-delà d'illustrer l'actualité de ces questions, les quelques références indiquées dans les questions de recherche pourront servir comme autant de repères dans ce chemin qu'il reste à découvrir. 
une nécessité. De la même manière que certaines recherches s'appuient sur la trajectoire socioprofessionnelle d'acteurs pour mettre en perspective leurs actions (Detchessahar 2003) ou le concept de "travail identitaire " (identity work) (Johnson, et al. 2010), d'autres chercheurs en management (à titre d'exemples Gardner et Martinko 1996, Hough et Ogilvie 2005) étudient les perceptions des acteurs en recourant pour validation à l'indicateur des types Myers-Briggs (MBTI) basé sur la théorie des types psychologiques de Carl Jung (1959), qui permet de décrire des profils managériaux. Selon la théorie des types psychologiques de Carl Jung (1959), les individus ont des préférences concernant quatre dimensions ou activités (deux modes étant possibles pour chaque dimension) : leur orientation de l'énergie (Extraversion/ Introversion), leur perception de la réalité (Sensation/Intuition), leur façon de prendre les décisions (pensée-Thinking/sentiment/Feeling) et leur style de vie (Perception/ Jugement). Tous les individus utilisent ces différents modes sur une base régulière, néanmoins ils ont une préférence pour un mode concernant chaque activité. Ces quatre paires de préférences se combinent pour former une typologie de 16 types distincts, les quatre préférences d'un individu indiquant son type. Aux différents types MBTI sont associés des profils managériaux (Krebs, Hirsh et Kummerow 2001) éclairant la compréhension de leurs pratiques. Le MBTI, comme d'autres outils psychologiques, s'il devait être retenu pour approfondir le lien acteur/pratique, devra toutefois être mobilisé avec prudence tant il vise à proposer une représentation figée de l'homme au travail difficilement compatible avec l'identité narrative précédemment évoquée et qui à l'inverse se définit par son caractère de permanente reconstruction. Ainsi, le recours à l'auto-confrontation croisée proposée par Clot (2005), comme source d'un développement de l'activité professionnelle, peut constituer une démarche méthodologique pertinente pour qui cherche à comprendre la relation entre la dynamique transformatrice de l'activité sur les acteurs et inversement. Parce quelle repose sur une " coanalyse qui mise sur un développement aussi bien du sujet, du collectif que de la situation" (Clot, et al. 2001: 18) des chercheurs avec les protagonistes, les entretiens en auto-confrontation croisée permettent d'ouvrir le champ des possibles de l'activité pour et par les acteurs eux-mêmes. Enfin, la conduite de ce programme de recherche (pour sa dimension communicationnelle) implique de mobiliser de nouvelles méthodologies d'analyse d'interactions et de conversations (Fairhurst et Cooren 2004, Fauré et Arnaud 2014), afin par exemple de mettre en lumière les mouvements rhétoriques identifiés par Samra-Fredericks (2003: 166) ${ }^{7}$ et d'approfondir la notion de compétence discursive des managers (Fauré et Rouleau 2011), et au-delà des participants à l'activité, comme autant de leviers d'action au service de la compétence collective.

7 Article dans lequel elle montre comment la compréhension/maîtrise de l'histoire de l'entreprise permet aux managers de sélectionner des moments spécifiques et de leur donner une signification ici et maintenant en lien avec leurs propres agendas personnels. 


\section{BIBLIOGRAPHIE}

ALVESSON M. et KARREMAN D., 2000, «Taking the linguistic turn in organizational research», Journal of Applied Behavioral Science, 36: 2, p. 136-158.

AMHERDT C.-H., DUPUICH-RABASSE F., EMERY Y. et GIAUQUE D., 2000, Compétence collective dans les organisations. Emergence, gestion et développement, Montréal, Les Presses de l'Université de Laval, 261.

ARGYRIS C. et SCHON D. A., 2002, Apprentissage organisationnel. Théorie, méthode, pratique, Paris Bruxelle, De Boeck-Wesmael, 380 p.

ARNAUD N., 2011, "L'ouverture du stock de connaissances de la compétence collective. Etude d'une conversation ", Finance Contrôle Stratégie, 14: 2, p. 101-141.

ARNAUD N.et MILLS C.E., 2012, «Understanding the inter-organizational agency: A communication perspective», Group and Organization Management, 37: 4, p. 452-485.

BAKKE J. W. et BEAN C. J., 2006, "The materiality of sensemaking», Tamara: A Journal of Critical Postmodern Organization Science, 5: 3, p. 51-69.

BARLEY S. R. et KUNDA G., 2001, «Bringing work back in», Organization Science, 12: 1, p. 76-95.

BATAILLE F., 2001, "Compétence collective et performance», Revue de Gestion des Ressources Humaines: 40, p. 66-81.

BERGER P. et LUCKMAN T., 1966, La construction sociale de la réalité, Paris, Armand Colin.

BIENCOURT O. et JOLIVET T., 2000, «Quand le marché interne devient conversationnel : conception des produits et modification des règles", Revue d'Économie Industrielle, $93: 4$, p. 51-73.

BIRCH C. L., 2000, The whole story handbook: Using imagery to complete the story experience, Little Rock, AR, August House, 128 p.

BODEN D., 1990, «The world as it happens: Ethnomethodology and conversation analysis», in G. RITZER (coord.), Frontiers of social theory: The new syntheses, New York, Columbia University Press, 1990, p. 185-213.

BODEN D., 1994, The business of talk: Organizations in action, Cambridge, Polity Press.

BOJE D. M., 1991, «The storytelling organization: A study of story performance in an office-supply firm.», Administrative Science Quarterly, 36: 1, p. 106-126.

BOJE D. M., LUHMAN J. T. et BAACK D. E., 1999, «Hegemonic stories and encounters between storytelling organizations», Journal of Management Inquiry, 8: 4, p. 340-360.

BOJE D. M. et SMITH R., 2010, «Re-storying and visualizing the changing entrepreneurial identities of Bill Gates and Richard Branson», Culture Eซ Organization, 16: 4, p. 307-331.

BOREHAM N., 2004, "A theory of collective competence: challenging the neoliberal individualisation of performance at work», British Journal of Educational Studies, 52: 2 , p. 5-17.

BOURDIEU P., 1980, Le sens pratique, Paris, Les Éditions de Minuit.

BOURDIEU P., 1986, "Lillusion biographique ", Actes de la recherche en sciences sociales, $62: 1$, p. $69-72$. 
BOUTET J., 2001, « Les mots du travail », in A. BORZEIX et B. FRAENKEL (coord.), Langage et travail. Communication, cognition, action, Paris, CNRS Éditions, 2001, p. 189-2003.

BRAY J., LEE J., SMITH L. et YORKS L., 2000, Collaborative inquiry in practice, Thousand Oaks, CA, SAGE.

BROWN A. D., 2006, «A Narrative Approach to Collective Identities», Journal of Management Studies, 43: 4, p. 731-753.

BROWN J. S. et DUGUID P., 2001, «Knowledge and organization: A social-practice perspective», Organization science, 12: 2, p. 198-213.

BROWN J. S. et DUGUID P., 2002, The social life of information, Boston, MA, Harvard Business Press, 356 p.

BURGOYNE J., 1989, «Creating the Managerial Portfolio: Building On Competency Approaches To Management Development», Management Learning, 20: 1, p. 56-61.

CAROLY S. et CHOLEZ C., 2007, "Compétences individuelles et compétences collectives: regard croisé entre sociologie et ergonomie», in W. CAVESTRO, C. DURIEUX et S. MONCHATRE (coord.), Travail et reconnaissance des compétences, Paris, Economica, 2007, p. 31-45.

CARROLL B., LEVY L. et RICHMOND D., 2008, «Leadership as practice: Challenging the competency paradigm», Leadership, 4: 4, p. 363-379.

CAVESTRO W., COLIN T. et GRASSER B., 2007a, «La gestion des compétences à l'épreuve de la compétence collective», in W. CAVESTRO, C. DURIEUX et S. MONCHATRE (coord.), Travail et reconnaissance des compétences, Paris, Economica, 2007a, p. 15-30.

CHÉDOTEL F., 2004, «Avoir le sentiment de faire partie d'une équipe: de l'identification à la coopération», $M @ n @ g e m e n t, 7: 3$, p.161-193.

CHÉDOTEL F. et PUJOL L., 2009, «De la compétence individuelle à la compétence collective : l'influence des processus d'identification social et le rôle du manager», in T. PICQ et C. DEFÉLIX (coord.), Gestion des compétences : Nouvelles relations, nouvelles dimensions, Paris, Vuibert, 2009, p. 79-99.

CHÉDOTEL F. et PUJOL L., 2012, « L'influence de l'identité sur la compétence collective lors de prises de décisions stratégiques ", Finance Contrôle Stratégie, 15: 1/2.

CLOT Y., 2005, L'autoconfrontation croisée en analyse du travail: l'apport de la théorie bakbtinienne du dialogue, Louvain-la-Neuve, Peeters, 37-55.

CLOT Y., FAÏTA D., FERNANDEZ G. et SCHELLER L., 2001, « Entretiens en autoconfrontation croisée: une méthode en clinique de l'activité », Education Permanente, 146: p. 17-27.

COLIN T. et GRASSER B., 2009, «Des compétences individuelles à la compétence collective : les apports d'une lecture en terme d'apprentissage dans un service d'urgence hospitalier", in T. PICQ et C. DEFÉLIX (coord.), Gestion des compétences : Nouvelles relations, nouvelles dimensions, Paris, Vuibert, 2009, p. 59-78.

COOREN F., 2000, The organizing property of communication, Amsterdam \& Philadelphia, John Benjamins Pub Co, 272 pages. 
COOREN F., 2004, «The communication achievement of collective minding: Analysis of board meeting excerpts», Management Communication Quarterly, 17: 4, p. 517-551.

COOREN F., 2010, Action et Agency in Dialogue: Passion, incarnation and ventriloquism, Philadelphia, John Benjamins Publishing Company, 224 p.

COOREN F., FAIRHURST G. et HUËT R., 2012, «Why matter always matters in (organizational) communication», in P. M. LEONARDI, B. A. NARDI et J. KALLINIKOS (coord.), Materiality and Organizing: Social Interaction in a Technological World, Oxford, UK, University Press Oxford, 2012, p. 296-314.

COOREN F. et FAIRHURST G. T., 2004, «Speech timing and spacing: The phenomenon of organizational closure», Organization, 11: 6, p. 793-824.

COORENF., KUHNT., CORNELISSENJ.P.et CLARKT.,2011, «Communication, organizing and organization: An overview and introduction to the special issue», Organization Studies, 32: 9, p. 1149-1170.

COOREN F., TAYLOR J. R. et VAN EVERY E. J., 2006, Communication as organizing. Empirical and theoretical exploration in the dynamic of text and conversation, London, Lawrence Erblaum Associates, Inc., 248 p.

CORRADI G., GHERARDI S. et VERZELLONI L., 2010, «Through the practice lens: Where is the bandwagon of practice-based studies heading?», Management Learning, 41: 3, p. 265-283.

CZARNIAWSKA-JOERGES B., 1998, A narrative approach to organization studies, London, Sage, 96 p.

D’AVENI R. A., 1995, Hypercompetition, Paris, Vuibert, 448 p.

DAMERON S., LE J. K. et LEBARON C., 2015, «Materializing Strategy and Strategizing Materials: Why Matter Matters», British Journal of Management, 26: p. S1S12.

DEFORNELM.etLÉONJ.,2000, «L'analyse de conversation, del'ethnométhodologie à la linguistique interactionnelle », Histoire épistémologie langage, 22: 1, p. 131-155.

DEFÉLIX C., BOULAIRE M. L., MONTIES V. et PICQT., 2014, «La compétence collective dans le contexte de la globalisation du management : retrouver le lien avec la performance», @GRH, 11: 2, p. 31-50.

DETCHESSAHAR M., 1998, «L'homologie des trajectoires socioprofessionnelles des acteurs de la coopération interentreprises : un vecteur de confiance et de stabilité», Finance, Contrôle, Stratégie, 1: 2, p. 49-70.

DETCHESSAHAR M., 2003, «L'avènement de l'entreprise communicationnelle ? Outils, problèmes et politique d'accompagnement ", Revue Française de Gestion, 29: 142, p. $65-84$.

DETCHESSAHAR M., 2011, «Santé au travail. Quand le management n'est pas le problème, mais la solution...", Revue française de gestion, 214: 5, p. 89-105.

DETCHESSAHAR M., 2013, «Faire face aux risques psycho-sociaux: quelques éléments d'un management par la discussion ", Négociations: 1, p. 57-80.

DOWNING S., 2005, «The social construction of entrepreneurship: Narrative and dramatic processes in the coproduction of organizations and identities ", Entrepreneurship Theory and Practice, 29: 2, p. 185-204. 
DREW P. et HERITAGE J., 1992, Talk at work: Interaction in institutional settings, Cambridge, Cambridge University Press, 245 p.

DUBOIS M. et RETOUR D., 1999, « La compétence collective: validation empirique fondée sur les représentations opératoires de travail partagées ", Psychologie du Travail et des Organisations, 5: 2-1, p. 225-243.

FAIRHURST G.T.etCOORENF.,2004, «Organizationallanguage in use:interaction analysis, conversation analysis and speech acts schematic», in D. GRANT, C.HARDY, C. OSWICK et L. PUTMAN (coord.), The SAGE Handbook of Organizational Discourse, London, Sage Publication Ltd, 2004, p.131-152.

FAURÉ B. et ARNAUD N., 2014, La communication des organisations, Paris, Repères, La Découverte, 128.

FAURÉ B. et ROULEAU L., 2011, «The strategic competence of accountants and middle managers in budget making», Accounting, Organizations E Society, 36: 3, p. 167182.

FENTON C. et LANGLEY A., 2011, «Strategy as practice and the narrative turn», Organization Studies, 32: 9, p. 1171-1196.

GABRIEL Y., 2000, Storytelling in organizations: Facts, fictions, and fantasies, New York, Oxford University Press, 276 p.

GARDNER W. L. et MARTINKO M. J., 1996, «Using the Myers-Briggs Type Indicator to study managers: A literature review and research agenda», Journal of Management, 22: 1, p. 45.

GEIGER D., 2009, «Revisiting the concept of practice: Toward an argumentative understanding of practicing», Management Learning, 40: 2, p. 129-142.

GEPHART R. P., 1978, «Status degradation and organizational succession: An ethnomethodological approach", Administrative science quarterly, 23: 4, p. 553-581.

GIDDENS A., 1984, The constitution of society, Cambridge, Polity Press, 345 p.

GROSJEAN M. et LACOSTE M., 1999, Communication et intelligence collective : le travail à l'hopital, Paris, PUF, 240.

HARDY C., LAWRENCE T. B. et GRANT D., 2005, «Discourse and collaboration: The role of conversations and collective identity», Academy of Management Review, 30: 1, p. $58-77$.

HARDY C. et PHILLIPS N., 2004, «Discourse and power», in D. GRANT, C. HARDY, C. OSWICK, N. PHILIPPS et L. PUTNAM (coord.), The Sage handbook of organizational discourse, 2004, p. 299-316.

HATCHUEL A., 1994, «Apprentissage collectifs et activité de conception ", Revue Française de Gestion, 99: Aout-Septembre, p. 25-41.

HONORÉ L., 2002, "Transformation de la relation de travail, rupture de l'identité professionnelle et dynamique disciplinaire ", Revue de Gestion des Ressources Humaines: 43, p. 102-113.

HOUGH J. R. et OGILVIE D., 2005, «An Empirical Test of Cognitive Style and Strategic Decision Outcomes», Journal of Management Studies, 42: 2, p. 417-448.

JARZABKOWSKI P., 2005, Strategy as practice: An activity-based approach, London, Sage Publications Ltd, 235 p. 
JARZABKOWSKI P., BALOGUN J. et SEIDL D., 2007, «Strategizing: The challenges of a practice perspective», Human Relations, 60: 1, p. 5-27.

JARZABKOWSKI P. et SPEE A. P., 2009, «Strategy-as-practice: A review and future directions for the field», International Journal of Management Reviews, 11: 1, p. 69-95.

JOHNSON P., BALOGUN J. et BEECH N., 2010, «Researching strategists and their identity in practice: building "close-with" relationships», in D. GOLSORKHI, L. ROULEAU, D. SEIDL et E. VAARA (coord.), Cambridge handbook of strategy as practice, Cambridge, Cambridge University Press, 2010, p. 243-257.

JUNG C. G., READ H., FORDHAM M., ADLER G. et HULL R. F. C., 1959, The collected works of CG Jung, Routledge \& Kegan Paul, $176 \mathrm{p}$.

KOENIG G., 1994, «L'apprentissage organisationnel : repérage des lieux », Revue Française de Gestion, 97: p. 293-306.

KREBS HIRSH S. et KUMMEROW J. M., 2001, Introduction aux types psychologiques dans les organisations, Paris, Édition française, CPP, $456 \mathrm{p}$.

KROHMER C., Vers le management des compétences collectives : proposition pour le repérage et la mise en oeuvre. Le cas d'une entreprise du secteur de la chimie, Thèse en sciences de gestion, Université Pierre Mendès-France, Grenoble, 2005. Disponible sur Thèses.fr.

LAINE P. M. et VAARA E., 2007, «Struggling over subjectivity: A discursive analysis of strategic development in an engineering group», Human Relations, 60: 1, p. 29-58.

LATOUR B., 2002, La fabrique du droit. Une ethnographie du Conseil d'État, Paris, La Découverte, $319 \mathrm{p}$.

LE BOTERF G., 2008, Travailler efficacement en réseau: une compétence collective, Paris, Editions Eyrolles, 156 p.

LLEWELLYN N. et SPENCE L., 2009, «Practice as a members' phenomenon», Organization Studies, 30: 12, p. 1419-1439.

MARKUS M. L. et BENJAMIN R. I., 1997, «The magic bullet theory in IT-enabled transformation», Sloan Management Review, 34: 2, p. 55-68.

MICHAUX V., 2003, Compétence collective et système d'information. Cing cas de coordination dans les centres de contacts, Thèse en sciences de gestion, Université de Nantes. Disponible sur http://www.theses.fr/

MIDLER C., BOUDES T. et CHARUE-DUBOC F., 1997, "Formation et apprentissage collectif dans les entreprises: une expérience dans le domaine du management de projet », Revue International de Gestion, 22: 3, p. 45-61.

MIETTINEN R., SAMRA-FREDERICKS D. et YANOW D., 2009, «Re-turn to practice: An introductory essay», Organization Studies, 30: 12, p. 1309-1327.

MILLS C. E., 2009, «Making organizational communication meaningful: Reviewing the key features of a model of sensemaking about change communication», Australian Journal of Communication, 36: 2, p. 111-126.

MINBAEVA D., FOSS N. et SNELL S., 2009, «Bringing the knowledge perspective into HRM», Human Resource Management, 48: 4, p. 477-483.

MYERS I. B., MCCAULLEY M. H. et MOST R., 1985, Manual: A guide to the development and use of the Myers-Briggs Type Indicator, Consulting Psychologists Press Palo Alto, CA, 186 p. 
NICOLINI D., GHERARDI S. et YANOW D., 2003, Knowing in organizations: A practice-based approach, Armonk, NY, ME Sharpe Inc, $177 \mathrm{p}$.

NORDHAUG O. et GRENHAUG K., 1994, «Competences as resources in firm», The International Journal of Human Resources Management, 5: 1, p. 89-106.

ORLIKOWSKI W. J., 2002, «Knowing in practice: Enacting a collective capability in distributed organizing», Organization Science, 13: 3, p. 249-273.

PANTIN F., 2010, « Conduire l'internationalisation des PME: Un processus porté par les compétences de l'équipe dirigeante », Revue internationale PME: Économie et gestion de la petite et moyenne entreprise, 23: 3-4, p. 13-42.

PHILLIPS N.,LAWRENCE T.B. et HARDY C., 2004, «Discourse and institutions», Academy of Management Review, 29: 4, p. 635-652.

PROCTER S. et BURRIDGE M., 2008, «Teamworking and performance: the extent and intensity of teamworking in the 1998 UK Workplace Employee Relations Survey (WERS98)», International Journal of Human Resource Management, 19: 1, p. 153-168.

PUTNAM L. L. et PACANOWSKY M. E., 1983, Communication and Organizations. An Interpretive Approach, Newbury Park, CA, Sage, 256 p.

QUINN R.W. et WORLINE M. C., 2008, «Enabling Courageous Collective Action: Conversations from United Airlines Flight 93", Organization Science, 19: 4, p. 497.

RETOUR D., 2005, «Le DRH de demain face au dossier compétences », Management E Avenir, 4: 2, p. 187-200.

RETOUR D. et KROHMER C., 2006, « La compétence collective, maillon clé de la gestion des compétences », in C. DEFÉLIX, A. KLARSFELD et E. OIRY (coord.), Nouveaux regards sur la gestion des compétences, Paris, Vuibert, 2006, p. 149-183.

RICHEBÉ N., 2007, " La fabrique des compétences collectives: agencements, ressources et coopération ", in W. CAVESTRO, C. DURIEUX et S. MONCHATRE (coord.), Travail et reconnaissance des compétences, Paris, Economica, 2007, p. 46-60.

RICEUR P., 1990, Soi-même comme un autre, Paris, Seuil 424 p.

ROBICHAUD D., 2006, «Steps toward a relational view of agency», in F. COOREN, J. R. TAYLOR et E. VAN EVERY (coord.), Communication as organizing Mahwah, NJ, Lawrence Erlbaum, 2006, p. 101-114.

ROBICHAUD D., GIROUX H. et TAYLOR J. R., 2004, «The metaconversation: The recursive property of language as a key to organizing», Academy of Management Review, 29: 4, p. 617-634.

ROULEAU L. et BALOGUN J., 2011, «Middle managers, strategic sensemaking, and discursive competence», Journal of Management Studies, 48: 5, p. 953-983.

ROY M., 1999, « Les équipes semi-autonomes au Québec et la transformations des organisations ", Gestion, 24:3, p. 76-85.

SACKS H., SCHEGLOFF E. A. et JEFFERSON G., 1974, «A simplest systematics for the organization of turn-taking for conversation», Language, 50: 4, p. 696-735.

SAMRA-FREDERICKS D., 2003, «Strategizing as lived experience and strategists' everyday efforts to shape strategic direction», Journal of Management Studies, 40: 1, p. $141-174$. 
SCHATZKI T. R., KNORR-CETINA K. et VON SAVIGNY E., 2001, The practice turn in contemporary theory, New York, Routledge, $186 \mathrm{p}$.

TAYLOR J. R., 1993, Rethinking the theory of organizational communication: How to read an organization, Norwood, NJ, Ablex, $284 \mathrm{p}$.

TAYLOR J. R., 2006, «Coorientation : a conceptual framework», in F. COOREN, J. R. TAYLOR et E. J. VAN EMERY (coord.), Communication as organizing : empirical and theoretical exploration in the dynamic of text and conversation, New Jersey, Lawrence Erlbaum Associates, 2006, p. 141-157.

TAYLORJ.R. et COORENF., 1997, «What makes communication “organizational”? : How the many voices of a collectivity become the one voice of an organization ", Journal of Pragmatics, 27: 4, p. 409-438.

TRUC G., 2005, «Une désillusion narrative? De Bourdieu à Ricœur en sociologie », Tracés. Revue de Sciences humaines: 8, p. 47-67.

TSOUKAS H. et CHIA R., 2002, «On Organizational Becoming: Rethinking Organizational Change», Organization Science, 13: 5, p. 567-582.

TYLER J.A., 2007, «Incorporating storytelling into practice: How HRD practitioners foster strategic storytelling», Human Resource Development Quarterly, 18: 4, p. 559-587.

VAARA E., 2010, «Taking the linguistic turn seriously: Strategy as a multifaceted and interdiscursive phenomenon", in J. A. C. BAUM et J. LAMPEL (coord.), The Globalization of Strategy Research (Advances in Strategic Management), New York, Emerald Group Publishing Limited, 2010, p. 29-50.

VICKERS D. et FOX S., 2010, «Towards practice-based studies of HRM: an actornetwork and communities of practice informed approach», The International Journal of Human Resource Management, 21: 6, p. 899-914.

WEICK K. E. et ROBERTS K. H., 1993, «Collective mind in organizations: Heedful interrelating on flight decks», Administrative Science Quarterly, 38: p. 357-381.

WHITTINGTON R., 2006, "Completing the practice turn in strategy research», Organization Studies, 27: 5, p. 613-634.

WHITTINGTON R., 2011, "The practice turn in organization research: Towards a disciplined transdisciplinarity», Accounting, Organizations and Society, 36: 3, p. 183-186.

WITTORSKI R., 1997, Analyse du travail et production de compétences collectives, Paris, L'Harmattan, 239.

WITTORSKI R., 2000, «La production de compétences collectives par et dans l'analyse des pratiques professionnelles», Psychologie du Travail et des Organisations, 6: 3-4, p. 75-102.

WOODILLA J., 1998, «Workplace conversations: the text of organizing», in D. GRANT, T. W. KEENOY et C. OSWICK (coord.), Discourse and Organization, London, Sage, 1998, p. 31-50.

ZARIFIAN P., 2009, Le travail et la compétence : entre puissance et contrôle, Paris, Presses Universitaires de France. 
Résumé: En partant de la notion de compétence collective telle que théorisée par les travaux des chercheurs français et québécois, cet article de synthèse propose de les prolonger et de les approfondir, à l'aide des travaux internationaux en communication organisationnelle et de ceux issus du tournant pratique en organisations. Sur ces bases, ce papier discute des pistes de recherche visant à mieux comprendre les dynamiques de production, de transformation et de soutien de la compétence collective. Finalement, cet article de synthèse propose une définition intégrative de la compétence collective, identifie des pistes concrètes de recherches futures et des implications méthodologiques, et enfin souligne l'intérêt d'une telle approche pour étudier la compétence collective à différents niveaux des organisations.

Mots-clés: article de synthèse, compétence collective,communication organisationnelle, pratiques.

Abstract: Based on the concept of collective competence as theorized by the work of researchers from France and Québec, this article proposes to extend and complete them by using the international works in organizational communication as well as those from the practice turn in organizations. On this basis, the paper identifies and discusses new research directions to better understand how collective competence is produced, processed and can be better supported in organizations. Finally, this review article offers an integrative definition of the notion of collective competence, identifies concrete directions for future research and methodological implications, and finally emphasizes the importance of such an approach for studying the collective competence at different levels of organizations.

Keywords: review article, collective competence, organizational communication, practices. 